\title{
The Fuzzy Control of the Unknown Obstacles Avoidance for the Mobile Robots
}

\author{
Chih-Keng Chen*, Jian-Da Wu ${ }^{* *}$ and Chen-Yu Wang* \\ * Department of Mechanical Engineering,Da-Yeh University, ckchen@mail.dyu.edu.tw \\ ** Department of Vehicle Engineering,Da-Yeh University, jdwu@ mail.dyu.edu.tw
}

\begin{abstract}
ABSTRAC'T
In this paper, we investigate the path following control strategy for a mobile robot with unknown obstacles detected on the planned path. For a known space with known obstacles, the path planning methods can generate the shortest collision-free path from the start to the end. The unknown obstacles may appear on the planned path and the mobile may not follow the original path. The obstacle avoidance mode is added into the controller in the research. Fuzzy controllers with the five distance sensors on the mobile can guide the mobile successfully. The computer simulations are given to verify the proposed algorithm. We find that locations and directions of the distance sensors will affect the results.
\end{abstract}

\section{INTRODUCTION}

The motion of mobile robot is restricted by the nonholonomic constraint, which is a challenging problem in the control applications[2][5]. The basic problem of mobile robot motion is to guide the cart from the start to the destination without making any contact with the obstacles in the workspace[1]. In a known workspace, many motion-planning algorithms are available for planning the path of the mobile[7]. In addition to the contact-avoidance requirement, the motion efficiency (path length and energy consumption, etc.) is also considered[6].

The problems of path following control are studied to track the well-planned path. Nonlinear control approach with complicated mathematical efforts has been studied by many researchers[8][9]. Fuzzy logic controller does not require the mathematical model of the control system and is used in the following control problem. The Sugeno-type fuzzy controller is designed and the cart is controlled to the desired path[10]. Another approach[4] is to design the controller to emulate the human driver. The human-expert can guide the mobile to track the path by their experience. The driving actions from the human driver can be used as the training data to generate the appropriate fuzzy controller[1]. In this way, [3][9][11][12] the truck backer-upper control problem for mobile robot is studied.

If the locations and orientations of the obstacles in the workspace change, or additional unknown obstacles appear on the planned path, the mobile may not follow the original path. Therefore, the obstacle avoidance mode will be incorporated into the path-following controller. In this paper, two fuzzy controllers are used for the two control modes. We assume that the five distance sensors on the mobile can detect the distances between the obstacles and the mobile in five different directions. Based on the information of the known environment and feedback of the distance sensors, the controller will determine if the detected obstacles are in the known database or are not considered in the path planning stage. Once the unknown objects are detected during the path following, the controller will switch to the obstacle avoidance mode to circumambulate obstacles. This mode will continue until the unknown objects are bypassed. Then, the path following mode will take over again to follow the planned path to the goal. Computer simulations are given to verify the proposed algorithm.

\section{MODEL OF THE MOBILE ROBOT}

The mobile robot considered here in this paper is shown in Fig. 1.

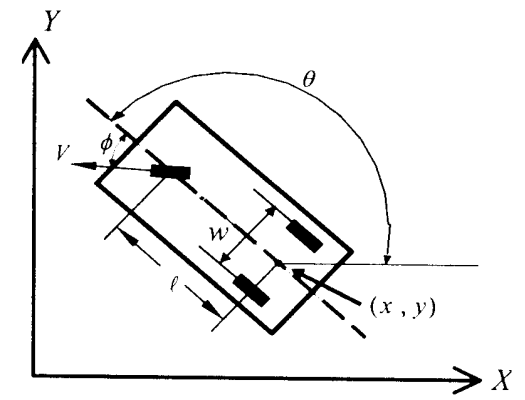

Fig. 1 The model of mobile robot 
The robot has three wheels that are assumed to have only rolling motion without slipping. The center of the front wheel is moving with a velocity $v$ and it is free to steer in the angle $\phi$ with respect to the cart. The distance between front and rear wheel axle is . The two rear wheels are separated by the $w$ distance and the coordinate of the middle point in the rear wheel axle is $(x$, $y$ ). The system has the nonholonomic constraint due to the non-slipping assumption in the rear wheels.

$$
\dot{x} \sin (\theta)-\dot{y} \cos (\theta)=0
$$

The equations of motion of the kinetic model are given in equation (1).

$$
\begin{aligned}
\dot{x} & =v \cos (\phi) \cos (\theta) \\
\dot{y} & =v \cos (\phi) \sin (\theta) \\
\dot{\theta} & =\frac{v}{\ell} \sin (\phi)
\end{aligned}
$$

\section{PATH FOLLOWING CONTROL}

For the path following control, the table look-up scheme is used for the controller design[12]. The algorithm can generate the fuzzy control rules by the training data. The training data are produced by emulating the driver to steer and control the mobile robot to track the desired path. We design the fuzzy system based on the input-output data pairs and replace the human driver by the designed fuzzy system.

\subsection{The training data}

We will consider the vertical path following problem first as shown in Fig. 2. The general oblique path tracking problem will also be studied later in this section based on the controller designed here.

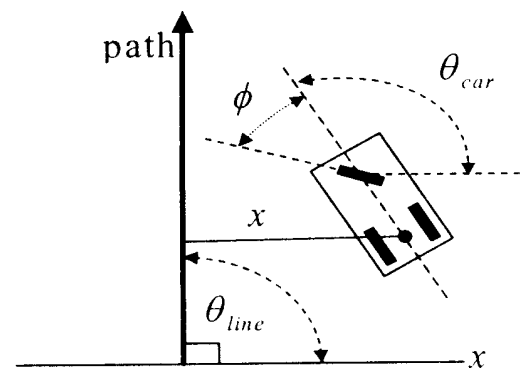

Fig. 2 The mobile robot following the vertical path

The car speed $v$ is assumed to be constant. In order to follow the vertical path, we adjust the steering angle $\phi$ to guide the car. In the study, we use the human experience (or common sense) to determine the control angle $\phi$ at every stage (i.e. for every pair of $x$ and $\theta_{c a r}$ ). By trial and error procedure, we can always drive the mobile robot to follow the desired path, i.e. $\left(x, \theta_{\text {car }}\right)$ will approach to $\left(0,90^{\circ}\right)$, in the smoothest trajectory.

During the simulation, we assume the range of the variables are $x \in[-10,10], \theta_{c a r} \in\left[-90^{\circ}, 270^{\circ}\right]$ and $\phi \in\left[-30^{\circ}, 30^{\circ}\right]$. The parameters of the system are assumed to be $\ell=1, w=0.5$ and $\nu=1$. Table 1 shows the data sequence for each time step from the initial position $\left(x, \theta_{\text {car }}\right)=\left(5,90^{\circ}\right)$. The trajectory of the mobile robot is simulated and plotted in Fig. 3. In the same way, the following 38 initial states were used to generate the desired input-output pairs: $\left(x, \theta_{\text {car }}\right)=\left(-9,0^{\circ}\right),\left(-9,90^{\circ}\right)$, $\left(-9,270^{\circ}\right),\left(-7,0^{\circ}\right),\left(-7,90^{\circ}\right),\left(-7,180^{\circ}\right),\left(-7,270^{\circ}\right), \ldots,(7$, $\left.0^{\circ}\right),\left(7,90^{\circ}\right),\left(7,180^{\circ}\right),\left(7,270^{\circ}\right),\left(9,90^{\circ}\right),\left(9,180^{\circ}\right),(9$, $\left.270^{\circ}\right)$

Table 1 The trajectory $\left(x, \theta_{c a r}\right)$ and the used steering angle $\phi$ from the initial state $\left(5,90^{\circ}\right)$

\begin{tabular}{|c|c|c|}
\hline$x$ & $\theta_{\text {car }}$ & $\phi$ \\
\hline 5.0 & 90.0 & 20 \\
\hline 4.85 & 108 & 14.5 \\
\hline 4.68 & 115.1 & 11.6 \\
\hline 4.45 & 121 & 8.6 \\
\hline$\ldots \ldots \ldots$ & $\ldots \ldots \ldots$ & $\ldots \ldots$. \\
\hline 0 & 90 & 0 \\
\hline
\end{tabular}

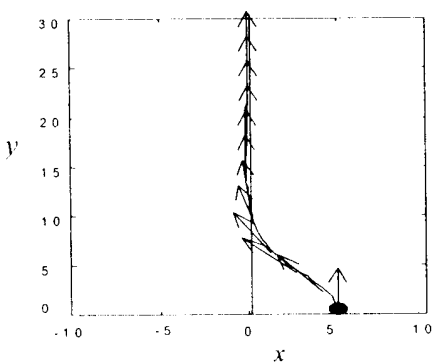

Fig. 3 The track of the mobile robot starting from $\left(5,90^{\circ}\right)$

\subsection{The table look-up scheme}

The fuzzy controller is constructed by a set of linguistic descriptions of the control rules. These rules can be determined by the direct expressions of the domain experts. On the other hand, the rules can also be extracted from demonstrations of the human experts. In this paper, we use the table look-up scheme to generate the rule table in the FIS(Fuzzy Inference System) based on the training data from the previous trial and error procedure [3][9][12].

The table look-up method uses the one training data to generate one if-then rule, and also the degree for the corresponding rule. If the rules generated do not conflict with any others, they will be kept in the rule table. If the 
rules are conflicting, i.e. the rules with the same If parts differ in the then parts, the one with the largest rule degree will be kept in the rule base. The following procedure shows the simplified table look-up scheme for a single input and single output system. The general procedure can be also found [12]. It is assumed that the obtained $N$ training data pairs are $\left(u^{p}, v^{p}\right), p=1, \ldots, N$, where $u$ is the input variable and $v$ is the output variable.

Step 1. Define the fuzzy sets to cover the input and output spaces

First, based on the maximum and minimum values in the training data, we can determine the range of each variable. That is the universe of discourse for each variable is $u \in\left[u_{\min }, u_{\max }\right]$ and $v \in\left[v_{\min }, v_{\max }\right]$. Three membership functions are designed to cover the corresponding space as shown in Fig. 4.
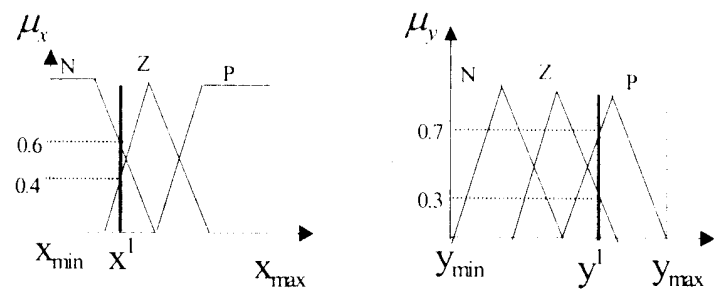

Fig. 4 The membership functions

Step 2. Compute the degree for each rule

Based on the membership functions defined in the previous step, the training data can be mapped from the crisp set to the fuzzy set. Each data pair can generate its corresponding rules. For example, if $\left(u^{l}, v^{1}\right)$ is one data pair from the training data set and the corresponding membership function values for the fuzzy sets are shown in Fig. 4. That is, $\mu_{N}\left(u^{1}\right)=0.6, \mu_{Z}\left(u^{1}\right)=0.4$, $\mu_{p}\left(v^{1}\right)=0.7$ and $\mu_{Z}\left(v^{1}\right)=0.3$. From the data pair, the following 4 linguistic rules will be proposed:

1. if $u^{1}$ is $N$ than $v^{1}$ is $P$
2. if $u^{1}$ is $N$ than $v^{1}$ is $Z$
3. if $u^{1}$ is $Z$ than $v^{1}$ is $P$
4. if $u^{1}$ is $Z$ than $v^{\prime}$ is $Z$

Among these rules, we can find that some rules have the same if parts but the different then part, e.g. rule 1 and 2 or rule 3 and 4 . The degree of a rule is then introduced to evaluate the conflicting rules. The degree of a rule is defined as follows:

$$
D(\text { rule })=\mu_{x}\left(u^{p}\right) \mu_{v}\left(v^{p}\right),
$$

as the rule is generated from the data pair $\left(u^{p}, v^{p}\right)$.

After the comparison of the degree for each conflicting rule, rule 1 and 3 are kept in the rule base, and their current degrees are stored for the further comparison when the conflict happen again.

\begin{tabular}{|c|c|}
\hline$D(1)=0.6 \times 0.7=0.42$ & $D(2)=0.6 \times 0.3=0.18$ \\
\hline$D(3)=0.4 \times 0.7=0.28$ & $D(4)=0.4 \times 0.3=0.12$ \\
\hline
\end{tabular}

Step 3. Construction of the rule base

After the $\mathrm{N}$ data pairs are substituted in to the table look-up scheme and the degrees comparison, the rule base of the fuzzy system can be generated.

After the previous training procedure, the fuzzy rule table can be listed as shown in Table 2. The fuzzy sets used in Table 2 are defined in Fig. 5.

Table 2 The fuzzy rule table

\begin{tabular}{|l|l|l|l|l|l|l|l|l|}
\hline \multicolumn{10}{|c|}{} & \multicolumn{8}{|c|}{$\theta_{c a r}$} \\
\cline { 2 - 9 } & S3 & S2 & S1 & CE & B1 & B2 & B3 \\
\hline & S2 & B2 & B1 & S1 & S2 & S3 & S3 & B2 \\
\hline & S1 & B3 & B2 & CE & S1 & S3 & S3 & B3 \\
\hline & CE & B3 & B3 & B2 & CE & S2 & S3 & S3 \\
\hline & B1 & S3 & B3 & B3 & B1 & CE & S2 & S3 \\
\hline & B2 & S2 & B3 & B3 & B2 & B1 & S1 & S2 \\
\hline
\end{tabular}
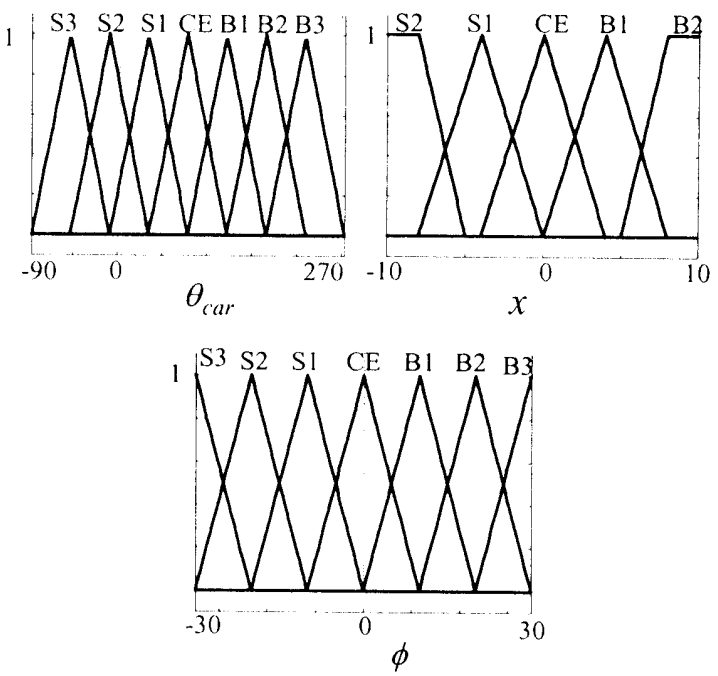

Fig. 5 The membership functions of each fuzzy set

Fig. 6 shows the trajectories of the mobile robot starting from the different initial states. In the figure, the vector is the direction of the car starting from the middle point of the rear wheel axle. The mobile is controlled by the fuzzy controller developed in the previous procedure. We can find that the same fuzzy system can also be used to control the mobile robot backward to track the vertical path by changing the sign of the input variables into the controller. When the mobile has approached the desired path, the rule to be fired in the rule table will be in the grey position in Table 2 . This means the steering angle will return to neutral 


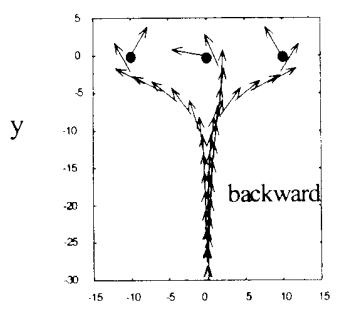

$\mathrm{X}$

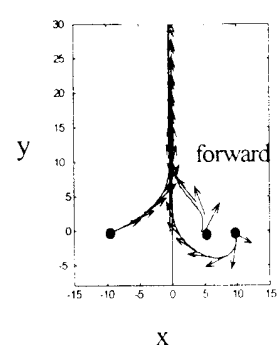

Fig. 6 The mobile robot from different initial states

\section{Oblique path following}

When the path direction $\theta_{\text {line }}$ changes from $90^{\circ}$ to another values, the previous fuzzy system in Table 2 can still be used to follow the oblique path. In this case, we have to replace the controller input from $\theta_{\text {cur }}$ to $\theta_{\text {car }}-\theta_{\text {line }}+90^{\circ}$ and the input $x$ to $d x$ as shown in Fig. 7. In this way, the controller developed can be used to follow the oblique path without any changes.

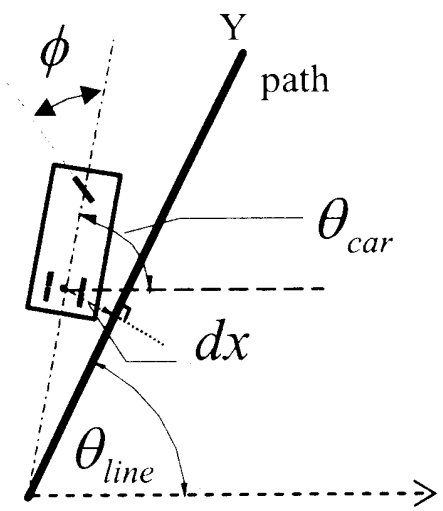

Fig. 7 The oblique path following

\section{UNKNOWN OBSTACLES AVOIDANCE}

The global path planning which considers both the minimization of path length and the collision free is done at the beginning[1]. During the path following control process, two control modes are used. The first one is the path following mode (mode I) for planned path as discussed in the previous section. The second one (mode II) is the obstacle-avoidance mode which will parry the unknown obstacle detected on the planned path. In this section, we discuss the control algorithm of the second mode by feedbacks of the distance sensors on the mobile robot and the information about the original workspace. We have the following assumptions for the system:

A. Three distance sensors separated by the included angle $\alpha$ are installed to measure the distance in directions of the front $\left(d_{c}\right)$, front right $\left(d_{r}\right)$ and front left $\left(d_{l}\right)$ for the robot. The sensors are assumed to give the linear distance between the obstacle and the reference point on the mobile robot.

B. The maximum distance the sensor can measure is $d_{\max }$. When the distance is larger than this value, i.e. $d>d_{\max }$, the sensor cannot give any signal.

C. To ensure that the mobile robot can pass through the obstacles without being trapped in, the spaces between any two obstacles are assumed to be larger enough to allow the robot to pass.

Distance sensors and the obstacle-avoidance logics

Based on the feedback signals, the steering angle can be determined by the following basic rules:

1. All the three sensors detect the obstacle

In the case of Fig. 8(a), if $d_{l}>d_{c}$ and $d_{l}>d_{r}$, the steering wheel should be turned left.

2. Two sensors detect the obstacle

In Fig. 8(b), if $d_{r}>d_{\max }$ and $d_{l}>d_{c}$, the steering wheel should be turned right.

3. Only one sensor detects the obstacle

In the case of Fig. 8(c), if $d_{l}$ and $d_{r}$ are greater than $d_{\max }$, the mobile robot can be turned left or right depending on the position information of the other known obstacles.

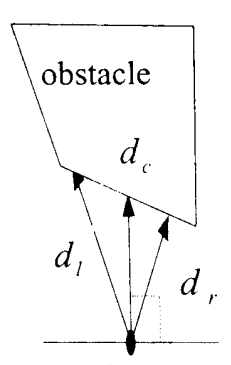

(a)

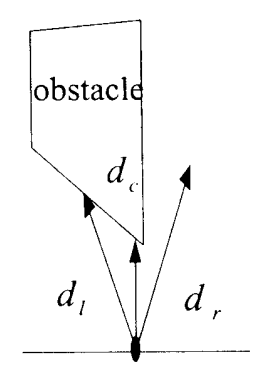

(b)

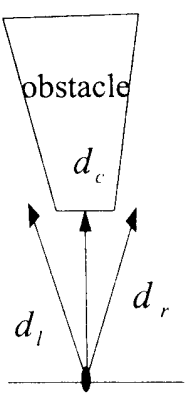

(c)
Fig. 8 Three cases when sensors detect the obstacle

The system block diagram is shown in Fig. 9. Five signals $d_{1}, d_{c}, d_{1}, \theta_{c a r}$ and $x$ are measured by the sensors. Using the information of $d_{1}, d_{c}, d_{r}$, the unknown obstacle can be detected for avoidance by the mode II control. If there is no unknown obstacle is scanned, the mobile robot will approach the closest planned path by switching the control mode back to mode I. The simulation result for the mobile robot with three distance sensors is shown in Fig. 10.

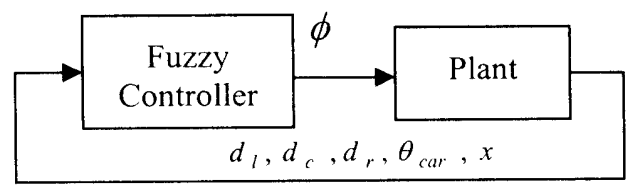

Fig. 9 The system block diagram 


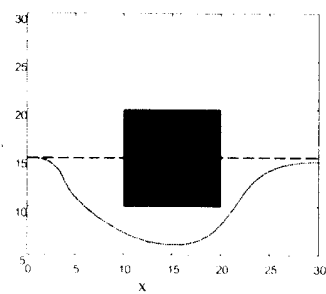

(a) The trajectory

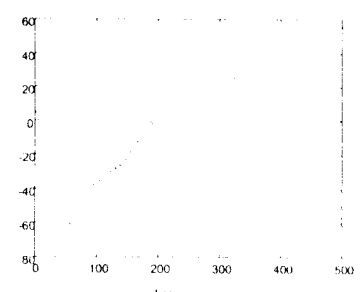

(b)Direction of robot
Fig. 10 Unknown obstacle avoidance with 3 distance sensors

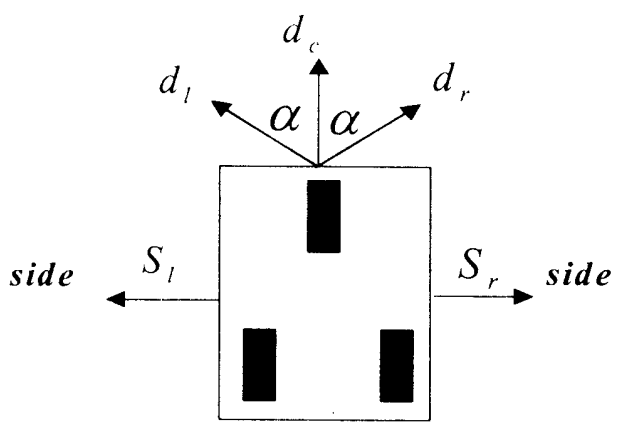

Fig. 11 Adding two side distance sensors

We first divide the sensors into two categories: front sensors and side sensors, and then determine the direction that mobile robot can pass the obstacles without collision. From the assumption $\mathrm{C}$ listed previously, the direction to parry the obstacles is between two obstacles. By the signals from the sensors, we use fuzzy logic rules to determine the new direction to follow. In this mode, the five distance variables are inputs to the fuzzy controller and the output variable is the angular change $\Delta \theta_{\text {lime }}$ to the direction angle of the original desired path. One configuration is shown as in Fig. 12.

The purpose of the mode II controller with five distance sensors is to determine the direction change $\Delta \theta_{\text {linc }}$. For example, when the car is on the path, and $d_{c}$ detects the unknown obstacle and one of $d_{l}, d_{r}$ detect the known obstacle, then the direction to go is between $d_{\text {c }}$ and $d_{l}$ or $d_{c}$ and $d_{,}$. The obstacle-avoidance direction $\bar{\theta}_{\text {line }}$ of the car is then represented as

$$
\bar{\theta}_{\text {line }}=\theta_{\text {line }}+\Delta \theta_{\text {line }}
$$

where $\theta_{\text {line }}$ is the original path direction and $\Delta \theta_{\text {lime }}$ is the direction change. The steering angle $\phi$ can be determined from the equation (1) by the following approximation.

$$
\dot{\theta}=\frac{\Delta \theta_{\text {lmee }}}{\Delta t}=\frac{v}{l} \times \sin (\phi)
$$

or

$$
\phi=\sin ^{-1}\left(\frac{t \times\left(\Delta \theta_{\text {line }}\right)}{V \times \Delta t}\right)
$$

\section{SIMULATION AND RESULTS}

In this section, we give the computer simulation of the path following and obstacle avoidance control for the mobile robot. Given the environments with known obstacles, the path planning algorithm 'center of nodes method' is used to generate the shortest path[1]. The fuzzy controller of mode $\mathrm{I}$ is utilized to follow the planned paths as seen in Fig. 13. We can find that the mobile robot can follow the path from the start to the end without any collision.

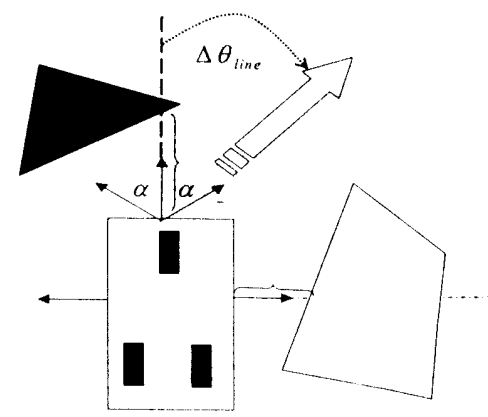

Fig. 12 The mobile robot detects the obstacles

Further, if the unknown obstacle is detected, i.e. the obstacle appears after the global path planning, the mode II controller will take over and guide the mobile robot to parry it. In Fig. 14, the dashed line is the originally planned path that is generated by considering the known obstacles only. One can find that the path following mode(mode I) will initially control the car to follow the planned path. Once the unknown obstacle (the solid blocks) is detected on the path, the controller will be switched to mode II and begin to parry the obstacle. Thus, the priority of mode II controller is higher than that of mode I. Since the information of the known obstacles are stored in the car, the controller will generate the path direction change $\Delta \theta_{\text {line }}$ in equation (2), and the mobile robot will follow the new direction by the same path following controller.
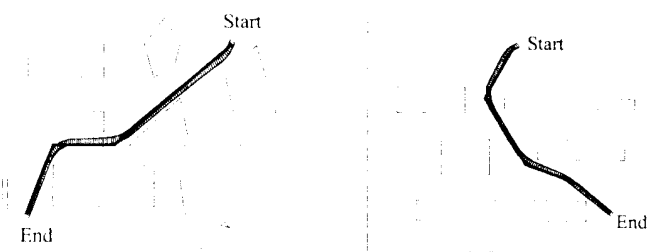

Fig. 13 Mobile Robot follows the planned path

During the mode II control, the mobile robot attempt to parry the obstacles on the planned path. Thus, the length of the real trajectory is greater than the original one. To compare the difference, the unknown obstacles are also considered in the path planning procedure to generate the new optimal path by doing the path planning again as shown in Fig. 14. In this case, the lengths of optimal new 
path and the real obstacle-avoidance path are subequal. However, we can find that the new path is straighter forward to the end without detour. Thus, if the online path planning is possible, it can generate the better path to follow.

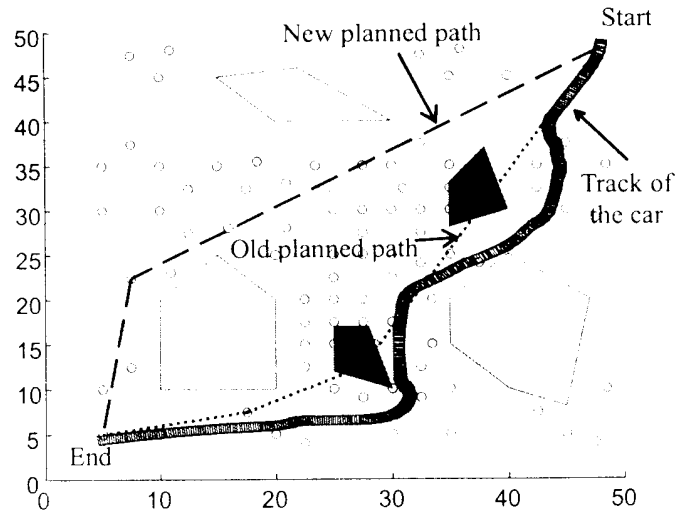

Fig. 14 Obstacle-avoidance control and the comparison of optimal new path and the real obstacle-avoidance path

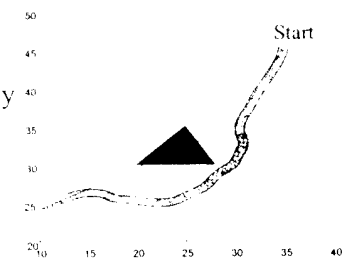

(a) $\alpha=45^{\circ}$

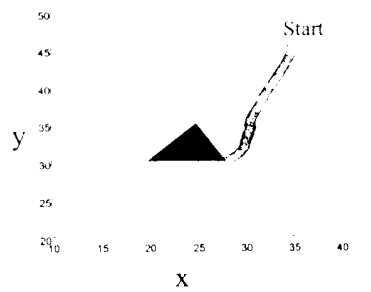

(b) $\alpha=20^{\circ}$
Fig. 15 Trajectories of the mobile robot with different $\alpha$

As mentioned in the previous section, the distance sensors are the important sources of the environmental information for the mobile robot. The orientation of the sensors may affect the control results and the ability to avoid the obstacles. In Fig. 15, we use two different included angles ( $\alpha$ in Fig. 11) for the front sensors and compare the difference in the obstacle-avoidance results at the same environment. From Fig. 15 (a) one can find that when $\alpha=45^{\circ}$ it can parry the obstacle successfully. However, if $\alpha=20^{\circ}$ is used for the sensors, the collision will happen as shown in Fig. 15 (b). This is due to the factor that angle between the front right and right side sensors are too large and thus cause the blind spot in the direction. This leads to the collisions since the mobile robot cannot detect the unknown obstacle in this direction. Therefore, the angle $\alpha$ should be increased to make the five sensors uniformly separated. If the unknown obstacles are of too many acute angles, then the number of sensors should be increased in order to reduce the blind spot from the sensors.

\section{CONCLUSION}

In this paper, we study the path following and obstacle -avoidance problem for the mobile robot from the starting states to the final one. The fuzzy controllers used in the two modes: path following (mode I) and obstacle avoidance (mode II) are different, and they are constructed and trained by the table look-up scheme with different training data. The computer simulations are implemented to show the results. From the results, we can find that for most paths with unknown obstacles of convex shape, the controller will steer the mobile robot to follow the desired path and avoid the obstacle respectively. We also find that the location and the direction of the distance sensors will affect the control result, and thus, this factor is also discussed in this paper.

\section{REFERENCES}

[1]C. K. Chen and C. Y. Wang, "The Path Following Fuzzy Control of Mobile Robot," Proceeding of $15^{\text {th }}$ National Conference on Mechanical Engineering CSME, Taiwan, 1998, pp. 415-422.

[2]J. Gomez Ortega and E.F Camacho "Mobile robot navigation in a partially structured static environment, using neural predictive control," Control Eng. Practice, Vol.4, No.12, 1996, pp.1669-1679.

[3]J. S. Jang, "ANFIS: Adaptive-Network-Based Fuzzy Inference System", IEEE Trans. on System, Man, and cybernetics, Vol.23, No.3, 1993, pp.665-685.

[4]Z. P. Jiang and N. Henk, "Tracking Control of Mobile Robots: A Case Study in Backstepping" Auotomatica. Vol.33, No.7, 1997, pp.1393-1399.

[5]H. Kolmanovsky and N. H. McClamroch, "Development in Nonholonomic Control Problems," IEEE Control Systems, 1995, pp.20-36.

[6]J. C. Latombe, Robot Motion Planning, Kluwer, Boston, 1991.

[7]Z. Li and J. F. Canny, editors, Nonholonomic Motion Planning, Kluwer, 1993

[8]R. M. Murray and S. S. Sastry, "Nonholonomic Motion Planning: Steering Using Sinusoids," IEEE Trans. on Automatic Control, Vol. 38, no. 5, pp. 700-716, 1993

[9]C. Samson, "Control of chained system: Application to path following and time-varying point-stabilization of mobile robots," IEEE Trans. on Automatic Control, Vol. 40, no. 1, pp. 64-77, 1995.

[10]K. Tanaka, M. Sano, "Trajectory stabilization of a model car via fuzzy control", Fuzzy sets and systems, 1995.

[11] L. X. Wang, A Course in Fuzzy System and Control, Prentice Hall, 1997.

[12] L. X. Wang, J. M. Mendel, "Generating Fuzzy Rules by Learning from 3 Examples," IEEE Trans. on System, Man, and cybernetics, Vol.22, No.6, pp. 1414-1427, 1992.

\section{ACKNOWLEDGEMENT}

The work was supported by the National Science Council in Taiwan, Republic of China, under the project number NSC89-2745-P-212-003. 